\title{
Limiting Behaviour of Dirichlet Forms for Stable Processes on Metric Spaces
}

by

\author{
Katarzyna PIETRUSKA-PAŁUBA
}

Presented by Stanisław KWAPIEŃ

Summary. Supposing that the metric space in question supports a fractional diffusion, we prove that after introducing an appropriate multiplicative factor, the Gagliardo seminorms $\|f\|_{W^{\sigma, 2}}$ of a function $f \in L^{2}(E, \mu)$ have the property

$$
\frac{1}{C} \mathcal{E}(f, f) \leq \liminf _{\sigma / 1}(1-\sigma)\|f\|_{W^{\sigma, 2}} \leq \limsup _{\sigma / 1}(1-\sigma)\|f\|_{W^{\sigma, 2}} \leq C \mathcal{E}(f, f),
$$

where $\mathcal{E}$ is the Dirichlet form relative to the fractional diffusion.

1. Inroduction. For $f \in L^{p}\left(\mathbb{R}^{d}\right), 0<\sigma<1, p>1$, consider the so-called Gagliardo seminorm of $f$ :

$$
\|f\|_{W^{\sigma, p}(\Omega)}=\left(\iint_{\Omega} \frac{|f(x)-f(y)|^{p}}{|x-y|^{d+\sigma p}} d x d y\right)^{1 / p},
$$

where $\Omega$ is a connected open subset of $\mathbb{R}^{d}$. The restriction to $\sigma<1$ is mandatory: when $\sigma \geq 1$, then the finiteness of (1.1) results in $f$ being a constant function (see e.g. [8]). The seminorm (1.1) is the intrinsic seminorm in the fractional Sobolev space $W^{\sigma, p}(\Omega)$ (see [1, par. 7.43]). We are interested in the behaviour of (1.1) as $\sigma$ approaches the critical value $\sigma=1$. Bourgain, Brézis and Mironescu in [6], and further in [7], established the relation

$$
\lim _{\sigma \nearrow 1}(1-\sigma)\|f\|_{W^{\sigma, p}(\Omega)}^{p}=C_{p} \int_{\Omega}|\nabla f|^{p} d x=C_{p}\|f\|_{W^{1, p}(\Omega)}^{p}
$$

( $\Omega$ is a smooth bounded domain in $\mathbb{R}^{d}, f \in W^{1, p}(\Omega), p>1$ ). Note that the

2000 Mathematics Subject Classification: Primary 60J35; Secondary 46E35.

Key words and phrases: Gagliardo seminorm, stable processes, metric spaces.

Partially supported by a KBN grant no. 1 PO3A 00829. 
meaning of $\|\cdot\|_{W^{\sigma, p}(\Omega)}$ is different for $\sigma<1$ and for $\sigma=1$, which is annoying but consistent with traditional notation. For the special case $p=2, \Omega=\mathbb{R}^{d}$, (1.2) follows from the previous work of Maz'ya and Nagel [18].

From another perspective, in this case $\left(\Omega=\mathbb{R}^{d}, p=2, \alpha<1\right)$ the expression

$$
\mathcal{E}^{(\alpha)}(f, f)=C_{\alpha} \iint_{\mathbb{R}^{d}} \frac{(f(x)-f(y))^{2}}{|x-y|^{d+2 \alpha}} d x d y \quad\left(=\|f\|_{W^{\alpha, 2}}^{2}\right)
$$

(with domain $\mathcal{D}\left(\mathcal{E}^{(\alpha)}\right)=W^{\alpha, 2}\left(\mathbb{R}^{d}\right)$ ) is the Dirichlet form of the subordinated symmetric $2 \alpha$-stable process on $\mathbb{R}^{d}$, while the Dirichlet integral

$$
\mathcal{E}(f, f)=\int_{\mathbb{R}^{d}}|\nabla f|^{2} d x \quad\left(=\|f\|_{W^{1,2}}^{2}\right),
$$

with domain $W^{1,2}$, is the Dirichlet form of the Brownian motion, and therefore the relation (1.2) asserts that the Dirichlet form of the Brownian motion on $\mathbb{R}^{d}$ can be recovered from the Dirichlet forms of stable processes.

In this note, we are concerned with a similar phenomenon arising for Brownian-like diffusions (fractional diffusions, see [2] for the definition) and related stable processes on metric measure spaces. Namely, suppose that $\mathcal{E}(f, f)$ is the Dirichlet form of the diffusion on a metric space $(E, \varrho)$ equipped with an Ahlfors $d$-regular measure $\mu$, and for $\alpha \in(0,1), \mathcal{E}^{(\alpha)}$ is the Dirichlet form of the subordinated $2 \alpha$-stable process. Then a similar statement holds: for any $f \in \mathcal{D}(\mathcal{E})$,

$$
\lim _{\alpha \nearrow 1} \mathcal{E}^{(\alpha)}(f, f)=\mathcal{E}(f, f) .
$$

Similarly to the classical case, we can consider the Gagliardo seminorms

$$
\|f\|_{W^{\sigma, 2}}=\left(\int_{E} \int_{E} \frac{(f(x)-f(y))^{2}}{\varrho(x, y)^{d+2 \sigma}} d \mu(x) d \mu(y)\right)^{1 / 2},
$$

which are now nontrivial up to $\sigma=d_{w} / 2, d_{w}$ being the walk dimension of $(E, \varrho, \mu)$ (in [20] it was proved that the finiteness of (1.4) with $\sigma \geq d_{w} / 2$ implies $f \equiv$ const). Since it is known (see Stós [21]) that the Dirichlet form of the $2 \alpha$-stable process compares to $\|f\|_{W^{\alpha d_{w}, 2}}^{2}$, our statement (1.3) obliges $\|f\|_{W^{\sigma, 2}}$ to tend to $\infty$ when $\sigma \nearrow d_{w} / 2$, and also, for $f \in \mathcal{D}(\mathcal{E})$,

$$
\begin{aligned}
\frac{1}{C} \mathcal{E}(f, f) \leq \liminf _{\alpha \nearrow 1}(1-\alpha) & \|f\|_{W^{\alpha d_{w} / 2,2}} \\
& \leq \limsup _{\alpha \nearrow 1}(1-\alpha)\|f\|_{W^{\alpha d_{w} / 2,2}} \leq C \mathcal{E}(f, f) .
\end{aligned}
$$

Since satisfactory differential techniques are unavailable in the setting of general metric spaces, our proof requires a different approach than the original one in $[6,7]$. 
2. Diffusion processes and their Dirichlet forms. Suppose that $(E, \varrho)$ is a separable, locally compact metric space and that $\mu$ is a Radon measure on $E$ such that

$$
C_{1} r^{d} \leq \mu(B(x, r)) \leq C_{2} r^{d}
$$

for some $d \geq 1$ and all $x \in E, r>0$ (i.e. the measure $\mu$ is Ahlfors $d$-regular). We require $E$ to satisfy the chain condition:

(C) for any $x, y \in E$ and $n \geq 1$ there exists a "chain" $x=x_{0}, x_{1}, \ldots, x_{n}=y$ such that $\varrho\left(x_{i}, x_{i+1}\right) \leq(C / n) \varrho(x, y)$ ( $C$ a universal constant $)$.

Further, assume that $(E, \varrho, \mu)$ supports a Markovian kernel $\{p(t, x, y)\}$, i.e. a family of measurable functions $p(t, \cdot, \cdot): E \times E \rightarrow \mathbb{R}_{+}, t>0$, which satisfies:

(M1) $p(t, x, y)=p(t, y, x)$ for all $t>0$ and $x, y \in E$ (symmetry),

(M2) $p(s+t, x, y)=\int_{E} p(s, x, z) p(t, z, y) d \mu(z)$ for all $s, t>0$ and $x, y \in E$ (the Chapman-Kolmogorov identity, or the Markov property),

(M3) $\int_{E} p(t, x, y) d \mu(y)=1$ for all $t>0$ and $x \in E$ (normalization),

(M4) $p(t, x, y)>0$ for all $t>0$ and $x, y \in E$ (irreducibility).

Our further assumption is that the Markovian kernel $p(t, x, y)$ satisfies the following estimate for all $t>0$ and $x, y \in E$ :

$$
\begin{aligned}
\frac{c_{1.1}}{t^{d / \beta}} \exp \left\{-c_{1.2}\left(\frac{\varrho(x, y)}{t^{1 / \beta}}\right)^{\beta /(\beta-1)}\right\} & \leq p(t, x, y) \\
\leq & \frac{c_{1.3}}{t^{d / \beta}} \exp \left\{-c_{1.4}\left(\frac{\varrho(x, y)}{t^{1 / \beta}}\right)^{\beta /(\beta-1)}\right\} .
\end{aligned}
$$

Examples of such spaces are the nested fractals ([2]) and other postcritically finite self-similar sets ([15]), Sierpiński carpets ([3]), and spaces that support the 2-Poincaré inequality $([22])$. It is known that the parameter $\beta$ does not depend on the particular kernel $p(\cdot, \cdot, \cdot)$, and is one of the characteristic constants of $(E, \varrho, \mu)$, called the walk dimension of $E$ and denoted by $d_{w}(E)$ (or just $d_{w}$ ). Under the chain condition $(\mathbf{C})$, it is known (see [2], [13]) that $2 \leq d_{w} \leq d+1$. For the Euclidean space $\mathbb{R}^{d}$, as well as other spaces supporting the 2-Poincaré inequality, the walk dimension is equal to 2, regardless of $d$. The walk dimension of the Sierpiński gasket in $\mathbb{R}^{d}$ is equal to $\log (d+3) / \log 2>2$. The exact value of the walk dimension for the Sierpiński carpet is unknown.

It has been proven lately in [14] that if we require the basic estimate (2.2) to be of the form $\left(c / t^{d / \beta}\right) \Phi\left(\varrho(x, y) / t^{1 / \beta}\right)$ with $\Phi:[0, \infty) \rightarrow[0, \infty)$ decreasing, then either $\Phi$ is an exponential function $\exp \left(-c s^{\beta /(\beta-1)}\right), \beta \geq 2$, and the corresponding Markov process is a diffusion, or it is equal to $1 /(1+s)^{d+\beta}$ and the process is not diffusive. 
Denote by $\left(P_{t}\right)_{t \geq 0}$ the semigroup of selfadjoint contraction operators on $L^{2}(E, \mu)$ associated with $\{p(t, x, y)\}$, given by

$$
L^{2}(E, \mu) \ni f(x) \mapsto P_{t} f(x)=\int_{E} p(t, x, y) f(y) d \mu(y) .
$$

We require the semigroup to be continuous at zero, i.e.

(M4) $\lim _{t \rightarrow 0^{+}} P_{t} f=f$ for all $f \in L^{2}(E, \mu)$, the limit taken in $L^{2}(E, \mu)$.

Such a strongly continuous semigroup on $L^{2}(E, \mu)$ gives rise to a Dirichlet form $\mathcal{E}$. There are several ways of defining it; the most convenient for our setting is the following (see [9], [11]). For $f \in L^{2}(E, \mu)$ set

$$
\mathcal{E}_{t}(f, f)=\frac{1}{t}\left\langle\left(f-P_{t} f\right), f\right\rangle_{L^{2}(E, \mu)} .
$$

Because of (M1) and (M3), we have

$$
\mathcal{E}_{t}(f, f)=\frac{1}{2 t} \int_{E} \int_{E}(f(x)-f(y))^{2} p(t, x, y) d \mu(x) d \mu(y) .
$$

By an easy application of the spectral theorem, for any given $f \in L^{2}$, the mapping $t \mapsto \mathcal{E}_{t}(f, f)$ is decreasing. Therefore we can set

$$
\begin{aligned}
\mathcal{D}(\mathcal{E}) & =\left\{f \in L^{2}(E, \mu): \sup _{t>0} \mathcal{E}_{t}(f, f)<\infty\right\}, \\
\mathcal{E}(f, f) & =\lim _{t \downarrow 0} \mathcal{E}_{t}(f, f) .
\end{aligned}
$$

Assuming (M1)-(M5) and (2.2), it has been shown (see [16], [19], [13]) that the domain of this Dirichlet form, $\mathcal{D}(\mathcal{E})$, is actually equal to the space

$$
\mathcal{L}=\operatorname{Lip}\left(d_{w} / 2,2, \infty\right)(E) .
$$

The definition of this space is the following. For $f \in L^{2}(E, \mu)$ and $n=$ $1,2, \ldots$, let

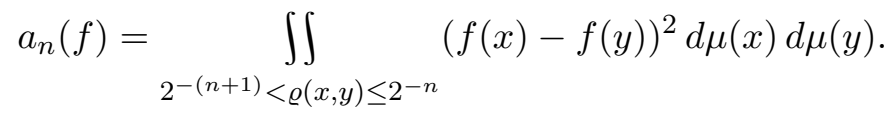

Then

$$
f \in \mathcal{L} \Leftrightarrow \sup _{n \geq 0}\left[2^{n\left(d+d_{w}\right)} a_{n}(f)\right]<\infty
$$

The norm in $\mathcal{L}$ is

$$
\|f\|_{\mathcal{L}}^{2}=\|f\|_{2}^{2}+\sup _{n \geq 0}\left[2^{n\left(d+d_{w}\right)} a_{n}(f)\right],
$$

and turns $\mathcal{L}$ into a Banach space. Also, there exists a universal constant $C$ such that for $f \in \mathcal{L}$ one has

$$
\frac{1}{C} \mathcal{E}(f, f) \leq \sup _{n \geq 0}\left[2^{n\left(d+d_{w}\right)} a_{n}(f)\right] \leq C \mathcal{E}(f, f) .
$$


Consider now the expression

$$
E^{(\alpha)}(f, f)=\iint_{E} \frac{(f(x)-f(y))^{2}}{\varrho(x, y)^{d+\alpha d_{w}}} d \mu(x) d \mu(y) .
$$

and introduce the following spaces $\Lambda_{\alpha}^{2,2}(E)$ :

$$
f \in \Lambda_{\alpha}^{2,2}(E) \Leftrightarrow E^{(\alpha)}(f, f)<\infty,
$$

with the norm

$$
\|f\|_{\Lambda_{\alpha}^{2,2}}^{2}=\|f\|_{2}^{2}+E^{(\alpha)}(f, f) .
$$

In the fractal setting, they were first considered in [21], and subsequently appeared in several articles. For a detailed account, see [12] or [17]. Note that these spaces have been known by the name of Besov-Slobodetskiı spaces. In [20], we have proven that for $\alpha \geq 1$, the finiteness of $E^{(\alpha)}(f, f)$ for a function $f \in L^{2}(E, \mu)$ results in $f \equiv$ const, and so the choice of the smoothness parameter $\alpha$ is restricted to $(0,1)$.

The following lemma is similar to its classical counterpart, nevertheless we give its proof for completeness.

Lemma 2.1. We have $\operatorname{Lip}\left(d_{w} / 2,2, \infty\right)(E) \subset \Lambda_{\alpha}^{2,2}(E)$. Moreover, this embedding is continuous,

$$
\|f\|_{\Lambda_{\alpha}^{2,2}} \leq C\|f\|_{\mathcal{L}}
$$

Proof. Take $f \in \mathcal{L}$. Split the integral defining $E^{(\alpha)}(f, f)$ into two parts: the first over the region $\varrho(x, y)>1$, the other over $\varrho(x, y) \leq 1$.

The first one is finite for any $f \in L^{2}$ : indeed,

$$
\begin{aligned}
\iint_{\varrho(x, y)>1} & \frac{(f(x)-f(y))^{2}}{\varrho(x, y)^{d+\alpha d_{w}}} d \mu(x) d \mu(y) \\
= & \sum_{n=0}^{\infty} \iint_{2^{n}<\varrho(x, y) \leq 2^{n+1}} \frac{(f(x)-f(y))^{2}}{\varrho(x, y)^{d+\alpha d_{w}}} d \mu(x) d \mu(y) \\
\leq & \sum_{n} \frac{1}{2^{n\left(d+\alpha d_{w}\right)}} \quad \iint_{2^{n}<\varrho(x, y) \leq 2^{n+1}}(f(x)-f(y))^{2} d \mu(x) d \mu(y) \\
\leq & 2 \sum_{n} \frac{1}{2^{n\left(d+\alpha d_{w}\right)}} \int_{2^{n}<\varrho(x, y) \leq 2^{n+1}}\left(f(x)^{2}+f(y)^{2}\right) d \mu(x) d \mu(y) \\
= & 4 \sum_{n} \frac{1}{2^{n\left(d+\alpha d_{w}\right)}} \quad \iint_{2^{n}<\varrho(x, y) \leq 2^{n+1}} f(x)^{2} d \mu(x) d \mu(y) \quad(\text { by symmetry) } \\
= & 4 \sum_{n} \frac{1}{2^{n\left(d+\alpha d_{w}\right)}} \int_{E} f(x)^{2} \mu\left(\left\{y: 2^{n}<\varrho(x, y) \leq 2^{n+1}\right\}\right) d \mu(x) \leq C\|f\|_{2}^{2}
\end{aligned}
$$

(in the last equality we used (2.1)). 
As to the remaining integral, we write

$$
\begin{aligned}
& \iint_{\varrho(x, y) \leq 1} \frac{(f(x)-f(y))^{2}}{\varrho(x, y)^{d+\alpha d_{w}}} d \mu(x) d \mu(y) \\
& \quad \leq \sum_{n=0}^{\infty} 2^{n\left(d+\alpha d_{w}\right)} \quad \iint_{2^{-(n+1)}<\varrho(x, y) \leq 2^{-n}}(f(x)-f(y))^{2} d \mu(x) d \mu(y) \\
& \quad \leq 2^{d+\alpha d_{w}} \sum_{n=0}^{\infty}\left[2^{n\left(d+d_{w}\right)} a_{n}(f)\right] \frac{1}{2^{n d_{w}(1-\alpha)}} \leq \frac{2^{d+\alpha d_{w}} 2^{d_{w}(1-\alpha)}}{2^{d_{w}(1-\alpha)}-1}\|f\|_{\mathcal{L}}
\end{aligned}
$$

and the continuity of the embedding is proven.

In particular, we see that all the spaces $\Lambda_{\alpha}^{2,2}(E), \alpha<1$, are dense in $L^{2}(E, \mu)$. This is so because $\mathcal{L}$, being the domain of the Dirichlet form of a Markov process, is in particular dense in $L^{2}(E)$.

\section{Stable processes and their Dirichlet forms}

3.1. Preliminaries on stable processes on metric spaces. The following definition of a stable process on a metric space supporting a fractional diffusion is taken from [5].

For a fixed parameter $\alpha \in(0,1)$, let $\left(\xi_{t}\right)_{t \geq 0}$ be the $\alpha$-stable subordinator, i.e. the process whose Laplace transform is given by $\mathbb{E} \exp \left(-u \xi_{t}\right)=$ $\exp \left(-t u^{\alpha}\right)$. Let $\eta_{t}(u), t>0, u \geq 0$, be its one-dimensional distribution density. For $t>0$ and $x, y \in X$ define

$$
p^{\alpha}(t, x, y)=\int_{0}^{\infty} p(u, x, y) \eta_{t}(u) d u .
$$

It is classical (see e.g. [4, p. 18]) that $p^{\alpha}(t, x, y)$ is the transition density of a Markov process, which we denote by $X^{\alpha}$ and call the symmetric $2 \alpha$-stable process on $E$. For further properties of this process and its transition density we refer the reader to [5].

From the property (see Th. 37.1 of [10])

$$
\lim _{u \rightarrow \infty} \eta_{1}(u) u^{1+\alpha / 2}=\frac{\alpha}{2 \Gamma(1-\alpha / 2)}
$$

and the scaling relation

$$
\eta_{t}(u)=t^{-2 / \alpha} \eta_{1}\left(t^{-2 / \alpha} u\right), \quad t, u>0,
$$

one deduces:

(P1) $\lim _{t \rightarrow 0} t^{-1} \eta_{t}(u)=(\alpha / \Gamma(1-\alpha)) u^{-1-\alpha}$ for $u>0$,

(P2) (formula (9) of [5]) $\eta_{t}(u) \leq c t u^{-1-\alpha}$ for $t, u>0$, 
(P3) (formula (10) of [5]) $\eta_{t}(u) \geq c t u^{-1-\alpha}$ for $t>0$ and $u>u_{0} t^{1 / \alpha}$, where $u_{0}=u_{0}(\alpha)$.

The Dirichlet form of the $2 \alpha$-stable process on $E$ is defined by $(2.3)-(2.5)$ and will be denoted by $\mathcal{E}^{(\alpha)}(f, f)$. In [21] it was proven that $\mathcal{D}\left(\mathcal{E}^{(\alpha)}\right)=$ $\Lambda_{\alpha}^{2,2}(E)$, and that there exists a universal constant $D=D(\alpha)$ such that

$$
\frac{1}{D} E^{(\alpha)}(f, f) \leq \mathcal{E}^{(\alpha)}(f, f) \leq D E^{(\alpha)}(f, f) .
$$

3.2. The main theorem. First, we prove the following theorem.

Theorem 3.1. Suppose $f \in \mathcal{D}(\mathcal{E})\left(=\operatorname{Lip}\left(d_{w} / 2,2, \infty\right)(E)\right)$. Then

$$
\lim _{\alpha \zeta 1} \mathcal{E}^{(\alpha)}(f, f)=\mathcal{E}(f, f) .
$$

Proof. In view of Lemma 2.1 and the characterization of the domain $\mathcal{D}\left(\mathcal{E}^{(\alpha)}\right), \mathcal{E}^{(\alpha)}(f, f)$ is well-defined. The explicit formula for $\mathcal{E}^{(\alpha)}$ is

$$
\begin{aligned}
\mathcal{E}^{(\alpha)}(f, f) & =\lim _{t \rightarrow 0} \frac{1}{2 t} \iint_{E} p^{\alpha}(t, x, y)(f(x)-f(y))^{2} d \mu(x) d \mu(y) \\
& =\lim _{t \rightarrow 0} \int_{0}^{\infty} \frac{1}{2 t}\left(\iint_{E} p(u, x, y)(f(x)-f(y))^{2} d \mu(x) d \mu(y)\right) \eta_{t}(u) d u \\
& =\frac{1}{2} \int_{0}^{\infty}\left(\int_{E} \int_{E} p(u, x, y)(f(x)-f(y))^{2} d \mu(x) d \mu(y)\right) \lim _{t \rightarrow 0} \frac{\eta_{t}(u)}{t} d u .
\end{aligned}
$$

To justify the last step (of putting the limit under the integral sign) we use the Lebesgue dominated convergence theorem: since for all $u, t>0$ we have $\eta_{t}(u) \leq c t u^{-1-\alpha}$ (property (P2)), the integrand in (3.3), being equal to $\frac{1}{2 t}\left\langle f-P_{u} f, f\right\rangle \frac{\eta_{t}(u)}{u}$, can be estimated by

$$
\frac{c}{u^{1+\alpha}}\left\langle f-P_{u} f, f\right\rangle \text {. }
$$

For large $u$ the contraction property of the semigroup yields

$$
(3.4) \leq \frac{c\|f\|_{2}^{2}}{u^{1+\alpha}}
$$

which is integrable for large $u$, and for small $u$ write

$$
(3.4)=\frac{2 c \mathcal{E}_{u}(f, f)}{u^{\alpha}} \leq \frac{2 c \mathcal{E}(f, f)}{u^{\alpha}},
$$

which in turn is integrable in the vicinity of 0 as long as $\alpha<1$.

Next, by (P1), one has

$$
\lim _{t \rightarrow 0} \frac{\eta_{t}(u)}{t}=\frac{\alpha}{\Gamma(1-\alpha)} \frac{1}{u^{1+\alpha}},
$$


and so by Fubini,

$$
\begin{aligned}
\mathcal{E}^{(\alpha)}(f, f) & =\frac{\alpha}{2 \Gamma(1-\alpha)} \iint_{E}\left(\int_{0}^{\infty} \frac{p(u, x, y)}{u^{1+\alpha}} d u\right)(f(x)-f(y))^{2} d \mu(x) d \mu(y) \\
& =\frac{\alpha}{\Gamma(1-\alpha)} \int_{0}^{\infty} \frac{1}{u^{\alpha}} \mathcal{E}_{u}(f, f) d u .
\end{aligned}
$$

It follows that for any fixed number $a>0$, any $\alpha \in(0,1)$, and any $f \in \mathcal{D}(\mathcal{E})$, as a result of the monotonicity of $\mathcal{E}_{u}(f, f)$,

$$
\begin{aligned}
\mathcal{E}^{(\alpha)}(f, f) & \geq \frac{\alpha}{\Gamma(1-\alpha)} \int_{0}^{a} \frac{1}{u^{\alpha}} \mathcal{E}_{u}(f, f) d u \geq \frac{\alpha}{\Gamma(1-\alpha)} \mathcal{E}_{a}(f, f) \int_{0}^{a} \frac{1}{u^{\alpha}} d u \\
& =\frac{\alpha}{\Gamma(1-\alpha)} \frac{a^{1-\alpha}}{1-\alpha} \mathcal{E}_{a}(f, f) .
\end{aligned}
$$

In particular, we can choose $a=1-\alpha$, which yields the estimate

$$
\mathcal{E}_{a}(f, f) \geq \frac{\alpha}{\Gamma(1-\alpha)} \frac{(1-\alpha)^{1-\alpha}}{1-\alpha} \mathcal{E}_{1-\alpha}(f, f) .
$$

Since $\lim _{t \rightarrow 0^{+}} t^{t}=1$ and $\lim _{t \rightarrow 0^{+}} \mathcal{E}_{t}(f, f)=\mathcal{E}(f, f)$, and $\lim _{t \rightarrow 0^{+}} t \Gamma(t)=1$, we obtain

$$
\liminf _{\alpha \nearrow 1} \mathcal{E}^{(\alpha)}(f, f) \geq \mathcal{E}(f, f) .
$$

The matching upper bound is simpler: this time around, write the integral (3.5) as

$$
\begin{aligned}
\mathcal{E}^{(\alpha)}(f, f) & =\frac{\alpha}{\Gamma(1-\alpha)}\left(\int_{0}^{1} \frac{1}{u^{\alpha}} \mathcal{E}_{u}(f, f) d u+\int_{1}^{\infty} \frac{1}{u^{\alpha}} \mathcal{E}_{u}(f, f) d u\right) \\
& =: \frac{\alpha}{\Gamma(1-\alpha)}\left(I_{1}+I_{2}\right) .
\end{aligned}
$$

These integrals are dealt with separately: $I_{1}$ will give the proper asymptotics, and $I_{2}$ will be negligible.

More precisely, since $\mathcal{E}_{u}(f, f) \rightarrow \mathcal{E}(f, f)$ and the limit is increasing, one has

$$
I_{1} \leq \mathcal{E}(f, f) \int_{0}^{1} \frac{d u}{u^{\alpha}}=\frac{\mathcal{E}(f, f)}{1-\alpha} .
$$

The integral $I_{2}$ can be rewritten as

$$
I_{2}=\int_{1}^{\infty} \frac{1}{u^{1+\alpha}}\left\langle f-P_{u} f, f\right\rangle d u
$$


and since the $P_{u}$ 's are contractions, $\left|\left\langle f-P_{u} f, f\right\rangle\right| \leq 2\|f\|_{2}^{2}$. Therefore

$$
\left|I_{2}\right| \leq 2\|f\|_{2}^{2} \int_{1}^{\infty} \frac{d u}{u^{1+\alpha}}=\frac{2}{\alpha}\|f\|_{2}^{2},
$$

and so

$$
\mathcal{E}^{(\alpha)}(f, f) \leq \frac{\alpha}{\Gamma(1-\alpha)(1-\alpha)}\left(\mathcal{E}(f, f)+\frac{2}{\alpha}(1-\alpha)\|f\|_{2}^{2}\right),
$$

giving

$$
\limsup _{\alpha / 1} \mathcal{E}^{(\alpha)}(f, f) \leq \mathcal{E}(f, f)
$$

As a corollary, we obtain

TheOREM 3.2. Suppose that $\mathcal{E}$ is the Dirichlet form associated with a fractional diffusion on $(E, \varrho, \mu)$ and let $E^{(\alpha)}(f, f), \alpha \in(0,1)$, be defined by (2.7). Then for any $f \in \operatorname{Lip}\left(d_{w} / 2,2, \infty\right)(E)$ we have

$$
\begin{aligned}
\frac{1}{C} \mathcal{E}(f, f) \leq \liminf _{\alpha / 1}(1-\alpha) E^{(\alpha)}(f, f) & \\
& \leq \limsup _{\alpha / 1}(1-\alpha) E^{(\alpha)}(f, f) \leq C \mathcal{E}(f, f)
\end{aligned}
$$

where the constant $C$ does not depend on $f$.

Proof. This follows from the representation (3.5) and the transition density estimate $(2.2)$.

Acknowledgements. The author wants to thank Jiaxin $\mathrm{Hu}$ and Andrzej Stós for valuable remarks.

\section{References}

[1] R. A. Adams, Sobolev Spaces, Academic Press, New York, 1975.

[2] M. T. Barlow, Diffusion on fractals, in: Lectures on Probability and Statistics, École d'Été de Probabilités de St. Flour XXV-1995, Lecture Notes in Math. 1690, Springer, New York, 1998, 1-121.

[3] M. T. Barlow and R. F. Bass, Brownian motion and analysis on Sierpinski carpets, Canad. J. Math. 51 (1999), 673-744.

[4] R. M. Blumenthal and R. K. Getoor, Markov Processes and Potential Theory, Pure Appl. Math., Academic Press, New York, 1968.

[5] K. Bogdan, A. Stós and P. Sztonyk, Harnack inequality for stable processes on d-sets, Studia Math. 158 (2003), 163-198.

[6] J. Bourgain, H. Brézis and P. Mironescu, Another look at Sobolev spaces, in: Optimal Control and PDE, In honour of Prof. A. Bensoussan's 60th birthday, J. L. Menaldi et al. (eds.), IOS Press, Amsterdam, 2001, 439-445.

[7] - - - - , Limiting embedding theorems for $W^{s, p}$ when $s \uparrow 1$ and applications, J. Anal. Math. 87 (2002), 77-101. 
[8] H. Brézis, How to recognize constant functions, Uspekhi Mat. Nauk, 57 (2002), no. 4 , 59-74 (in Russian); English transl.: Russian Math. Surveys 57 (2002), 693-708.

[9] E. A. Carlen, S. Kusuoka and D. W. Stroock, Upper bounds for symmetric Markov transition functions, Ann. Inst. Poincaré Probab. Statist. 23 (1987), 245-287.

[10] G. Doetsch, Introduction to the Theory and Application of the Laplace Transformation, Springer, New York, 1974.

[11] M. Fukushima, Dirichlet Forms and Markov Processes, Kodansha-North-Holland, 1980 .

[12] A. Grigoryan, Heat kernels and function theory on metric measure spaces, in: Contemp. Math. 338, Amer. Math. Soc., 2003, 143-172.

[13] A. Grigoryan, J. Hu and K. S. Lau, Heat kernels on metric measure spaces and an application to semilinear elliptic equations, Trans. Amer. Math. Soc. 355 (2003), 2065-2095.

[14] A. Grigoryan and T. Kumagai, On the dichotomy of the heat kernel two sidedestimates, in: Proc. Sympos. Pure Math. 77, Amer. Math. Soc., 2008, 199-210.

[15] B. M. Hambly and T. Kumagai, Transition density estimates for diffusion processes on post critically finite self-similar fractals, Proc. London. Math. Soc. 78 (1999), 431-458.

[16] A. Jonsson, Brownian motion on fractals and function spaces, Math. Z. 222 (1996), 495-504.

[17] T. Kumagai, Function spaces and stochastic processes on fractals, in: Fractal Geometry and Stochastics III, C. Bandt et al. (eds.), Progr. Probab. 57, Birkhäuser, 2004, 221-234.

[18] W. Masja [V. Maz'ya] und J. Nagel, Über äquivalente Normierung der anisotropen Funktionalräume $H^{\mu}\left(\mathbb{R}^{n}\right)$, Beiträge Anal. 12 (1978), 7-17.

[19] K. Pietruska-Pałuba, On function spaces related to fractional diffusions on d-sets, Stoch. Stoch. Rep. 70 (2000), 153-164.

[20] - Heat kernels on metric spaces and a characterization of constant functions, Manuscripta Math. 115 (2004), 389-399.

[21] A. Stós, Symmetric $\alpha$-stable processes on d-sets, Bull. Polish Acad. Sci. Math. 48 (2000), 237-245.

[22] K. T. Sturm, Diffusion processes and heat kernels on metric spaces, Ann. Probab. 26 (1998), 1-55.

Katarzyna Pietruska-Pałuba

Institute of Mathematics

University of Warsaw

Banacha 2

02-097 Warszawa, Poland

E-mail: kpp@mimuw.edu.pl

Received March 1, 2008;

received in final form July 28, 2008 